\title{
Dual-Loop Tube-based Robust Model Predictive Attitude Tracking Control for Spacecraft with System Constraints and Additive Disturbances
}

\author{
Runqi Chai, Member, IEEE, Antonios Tsourdos, Member, IEEE, Huijun Gao, Fellow, IEEE, \\ Yuanqing Xia, Senior Member, IEEE, and Senchun Chai, Senior Member, IEEE
}

\begin{abstract}
In this paper, the problem of optimal timevarying attitude tracking control for rigid spacecraft with system constraints and unknown additive disturbances is considered. Through the design of a new non-linear tubebased robust model predictive control (TRMPC) algorithm, a dual-loop cascaded tracking control framework is established. The proposed TRMPC algorithm explicitly considers the effect of disturbances and applies tightened system constraints to predict the motion of the nominal system. The obtained optimal control action is then combined with a non-linear feedback law such that the actual system trajectories can always be steered within a tube region centred around the nominal solution. To facilitate the recursive feasibility of the optimization process and guarantee the input-to-state stability of the tracking control process, the terminal controller and the corresponding terminal invariant set are also constructed. The effectiveness of using the proposed dual-loop TRMPC control scheme to track reference attitude trajectories is validated by experimental studies. A number of comparative studies were carried out, and the obtained results reveal that the proposed design is able to achieve more promising constraint handling and attitude tracking performance than that of the other newly developed methods investigated in this research.
\end{abstract}

Index Terms-Optimal time-varying attitude tracking, spacecraft, tube-based robust model predictive control, constraint tightening.

\section{INTRODUCTION}

\section{A. Literature Review}

$\mathbf{O}$ VER the last few decades, the design of an advanced attitude control system for rigid spacecraft has been recognized as a fundamental yet important issue. Since a well-established attitude control system is usually essential for stable (exo)atmospheric explorations and operations, it has received much attention from both academia and the aerospace industry [1], [2]. It is worth highlighting that attitude control systems are mainly developed to provide spacecraft with attitude stabilization or tracking capabilities. In terms of

R. Chai and A. Tsourdos are with the School of Aerospace, Transport and Manufacturing, Cranfield University, UK, e-mail: (r.chai@cranfield.ac.uk) and (a.tsourdos@cranfield.ac.uk).

$\mathrm{H}$. Gao is with the Research Institute of Intelligent Control and Systems, Harbin Institute of Technology, Harbin 150001, China, e-mail: (hjgao@hit.edu.cn).

Y. Xia and S. Chai are with the School of Automation, Beijing Institute of Technology, Beijing, China, e-mail: (xia_yuanqing@bit.edu.cn) and (chaisc97@163.com). attitude stabilization, many contributions have been reported thus far for completing this challenging task [3], [4]. For example, in [3], a composite attitude stabilization scheme was constructed by taking advantage of both disturbance observers and terminal sliding mode control (SMC). Similarly, the authors of [4] addressed the attitude stabilization problem of flexible spacecraft by proposing an adaptive fixed-time sliding mode controller.

In addition to attitude stabilization, another important task in space/aerospace vehicle control is to steer the attitude motion to track a pre-assigned reference trajectory. To achieve this goal, various robust tracking control algorithms have been developed in recent years with consideration of system uncertainties and environmental disturbances [5], [6]. Among these designs, SMC-oriented methods and finite-time control algorithms (and their extensions) have been recognized as two of the most promising candidates [7], [8]. Specifically, a reusable launch vehicle attitude tracking problem was studied in [7], where the authors designed and advocated a multivariable second-order SMC controller to ensure accurate tracking performance. In [8], the authors developed a distributed tracking algorithm for a group of rigid spacecraft in an uncertain environment. In their design, an adaptive neural-networkbased observer was combined with a distributed finite-time control scheme such that the dynamic leader can be robustly followed. However, most of these aforementioned investigations and algorithms did not fully consider the mechanical constraints, such as the state and input limitations, or simply assumed the controlled system to be unconstrained. This may result in poor tracking performance in engineering practice, where it is necessary to take system constraints into account. Furthermore, if unknown external disturbances exist in system equations, the state and input constraints tend to become more difficult to satisfy, thereby further degrading the control performance.

As an effective alternative, model predictive control (MPC) has the ability to deal with constrained systems. The advantage of applying MPC has been acknowledged in a number of literature works [9]-[11]. For example, Limon et al. [10] established a non-linear MPC scheme for tracking reference signals and successfully tested their design on an industrial four-tank process control platform. The authors of [11] investigated the reference tracking problem for autonomous underwater vehicles. In their research, a Lyapunov-based MPC scheme, equipped with a novel contraction constraint, was estab- 
lished to enhance the tracking performance. In the context of spacecraft or unmanned aerial vehicle (UAV) attitude control, some valuable MPC-based results have been reported [12][14]. For instance, the authors of [12] developed an explicit MPC scheme to control a linearized spacecraft model subject to system constraints. An important feature of their design is that the computational effort required by the algorithm is inexpensive, thereby making it suitable for systems with limited CPU resources. In addition, a spacecraft rendezvous and docking problem was considered in [13], wherein an MPC approach was successfully constructed and applied to control the linearized attitude dynamics. However, using a simplified dynamic model tends to result in infeasibility for practical systems. To address this issue, the authors of [15][17] investigated the modelling method for UAV trajectory tracking problems in the presence of external disturbances. In their works, various UAV system models such as the non-linear Hammerstein model and the autoregressive exogenous model were established and analysed. Moreover, in [18], the authors demonstrated that by installing a three-axis gimbal system on a UAV, the trajectory tracking performance of the MPC can be effectively improved. Aiming at optimizing the parameters used in the controller, in their follow-up work [19], the authors proposed and utilized a meta-heuristic swarm optimization algorithm.

Although the authors of the aforementioned works claimed that most of these proposed algorithms can achieve reliable attitude control performance, their conclusions are mainly supported by simulation or experimental studies. Rigorous mathematical analysis including the recursive feasibility and closed-loop stability continues to be a challenging problem. Note that in [20], the authors developed a non-linear MPC scheme to control the attitude of rigid spacecraft. In their research, the recursive feasibility and the closed-loop stability were guaranteed by constructing a homogeneous Lyapunov function and a locally stable terminal set. However, if the controlled system is perturbed by additive disturbances, these theoretical results may easily become invalid. Hence, deriving explicit conditions to guarantee these important properties is still difficult, especially for the non-linear robust model predictive attitude control scheme.

\section{B. Motivations and Contributions}

To actively deal with the disturbances or uncertainty existing in the operating environment, different robust MPC (RMPC) schemes have been proposed by researchers and engineers. One typical strategy is to apply a min-max structure. More specifically, the online optimization process considers all possible disturbance realizations, thereby obtaining a conservative robust solution [21], [22]. However, the computational complexity of this process is usually expensive and tends to significantly increase as the prediction horizon becomes wider. Another popular strategy is to use the so-called tube techniques [23], [24]. This strategy has been widely investigated for stabilizing linear systems. That is, the robust control law is made up of two parts: an optimized control policy and a feedback control policy. Here, the optimal control policy is responsible for steering the nominal states to the origin, whereas the feedback control policy is responsible for maintaining the actual state trajectory within a tube centred around the nominal system trajectory. Using this approach, several potential advantages such as enhanced robustness and guaranteed stability are likely to be obtained. However, a direct application of this strategy to the considered tracking control problem may be difficult due to the non-linear nature of the reference trajectory and attitude dynamics. Therefore, in this research, efforts are devoted to extend tube-based MPC (TMPC) such that it can be applied to address the considered problem. To be more specific, we aim to design and implement a non-linear tube-based robust MPC (TRMPC) scheme for rigid spacecraft such that the desired time-varying angular trajectories can be tracked with consideration of the system constraints and additive disturbances. In summary, different from other published works, the main contribution of this research lies in the following four aspects:

1) By developing a non-linear TRMPC algorithm, this work establishes a novel spacecraft attitude tracking control framework. The proposed design employs a dual-loop cascaded control structure and has the ability to track time-varying angular signals in the presence of disturbances and system constraints.

2) A compound feedback law, along with tightened state and control constraints, is designed such that robust constraint satisfaction can be achieved for all admissible disturbances.

3) Terminal controllers and the corresponding terminal invariant sets are established to facilitate the recursive feasibility of the TRMPC optimization process.

4) Explicit conditions are derived to guarantee the input-tostate stability (ISS) of the dual-loop tracking system and validated by a number of experimental studies.

To the best of the authors' knowledge, this paper provides the first attempt to design and implement a dual-loop TRMPC scheme with guaranteed feasibility and stability properties for rigid spacecraft attitude tracking control problems.

\section{Organization}

The remainder of this article is organized as follows. Sec II describes the non-linear attitude dynamics of spacecraft and the tracking control objective. Sec III presents the design of TRMPC for the outer- and inner-loop tracking systems, while some important theoretical properties are detailed in Sec IV. Following that, Sec V verifies the effectiveness of the proposed design by a number of experimental studies and analyses. Finally, concluding remarks are provided in Sec VI.

Notation: Let $\mathbb{R}^{n}$ and $\mathbb{R}$ stand for the $n$-dimensional Euclidean space and real space, respectively. The diagonal matrix is denoted as $\operatorname{diag}\left\{x_{1}, \ldots, x_{n}\right\}$, where $x_{1}, \ldots, x_{n}$ are the entries. For a vector $\mathbf{x}=\left[x_{1}, \ldots, x_{n}\right]^{T},|\mathbf{x}|=\left[\left|x_{1}\right|, \ldots,\left|x_{n}\right|\right]^{T}$ and $\|\mathbf{x}\|=\sqrt{\mathbf{x}^{T} \mathbf{x}}$. Similarly, $\|\mathbf{x}\|_{P}=\sqrt{\mathbf{x}^{T} \mathbf{P} \mathbf{x}}$ is the $\mathbf{P}$-weighted norm. Here, $\mathbf{P}$ is positive definite. For an $n$-by- $n$ matrix $\mathbf{Z}$, $\|\mathbf{Z}\|=\sqrt{\lambda_{\max }\left(\mathbf{Z}^{T} \mathbf{Z}\right)}$ stands for its 2-norm. Here, $\lambda_{\max }(\cdot)$ and $\lambda_{\min }(\cdot)$ represent the maximum and minimum eigenvalues, respectively. Consider two sets $\mathbb{A} \subset \mathbb{R}^{n}$ and $\mathbb{B} \subset \mathbb{R}^{n}$; we 
have the operations $\mathbb{A} \oplus \mathbb{B}=\{x+y \mid x \in \mathbb{A}, y \in \mathbb{B}\}$ and $\mathbb{A} \ominus \mathbb{B}=\left\{z \in \mathbb{R}^{n} \mid\{z\} \oplus \mathbb{B} \subset \mathbb{A}\right\}$.

\section{Problem Formulation}

\section{A. Spacecraft Attitude Dynamics}

Consider the attitude dynamics of a rigid spacecraft in the form of [9], [25], [26]:

$$
\begin{aligned}
& \dot{\boldsymbol{\Theta}}=\mathbf{R} \boldsymbol{\omega}+\boldsymbol{\Delta}_{f} \\
& \dot{\boldsymbol{\omega}}=-\mathbf{I}^{-1} \boldsymbol{\Omega} \mathbf{I} \boldsymbol{\omega}+\mathbf{I}^{-1} \mathbf{M}+\boldsymbol{\Delta}_{d}
\end{aligned}
$$

In Eq. (1), $\boldsymbol{\Theta}=[\alpha, \beta, \sigma]^{T}$ consists of three angular variables: angle of attack $\alpha$, sideslip angle $\beta$, and bank angle $\sigma \cdot \boldsymbol{\omega}=$ $[p, q, \nu]^{T}$ consists of three angular rate variables: roll rate $p$, pitch rate $q$ and yaw rate $\nu . \mathbf{M}=\left[M_{x}, M_{y}, M_{z}\right]^{T}$, where $M_{i}, i=(x, y, z)$ represents the roll, pitch and yaw moments. $\boldsymbol{\Delta}_{f} \in \mathbb{R}^{3}$ and $\boldsymbol{\Delta}_{d} \in \mathbb{R}^{3}$ stand for the additive disturbances due to environmental noise and model simplification. The matrix terms $\mathbf{R}, \mathbf{I}$, and $\Omega \in \mathbb{R}^{3 \times 3}$ are given by:

$$
\begin{gathered}
\mathbf{R}=\left[\begin{array}{ccc}
-\tan \beta \cos \alpha & 1 & -\tan \beta \sin \alpha \\
\sin \alpha & 0 & -\cos \alpha \\
-\cos \beta \cos \alpha & -\sin \beta & -\cos \beta \sin \alpha
\end{array}\right] \\
\mathbf{I}=\left[\begin{array}{ccc}
I_{x x} & 0 & -I_{x z} \\
0 & I_{y y} & 0 \\
-I_{x z} & 0 & I_{z z}
\end{array}\right], \boldsymbol{\Omega}=\left[\begin{array}{ccc}
0 & -\nu & q \\
\nu & 0 & -p \\
-q & p & 0
\end{array}\right]
\end{gathered}
$$

In Eq. (3), $I_{i j}(i, j=x, y, z)$ represents the inertia moments.

\section{B. Control Problem Objectives}

In this paper, two primary control objectives are expected to be achieved when designing the attitude controller:

- The actual attitude profiles $\Theta$ can be steered to follow the desired spacecraft attitude angle profiles $\Theta_{\mathrm{r}}=$ $\left[\alpha_{\mathrm{r}}, \beta_{\mathrm{r}}, \sigma_{\mathrm{r}}\right]^{T}$ in the presence of unknown disturbances $\boldsymbol{\Delta}_{f}$ and $\boldsymbol{\Delta}_{d}$.

- The tracking performance can be optimized while simultaneously satisfying the system state and control constraints.

For the first objective, if we define the tracking error vectors as $\mathbf{E}_{\theta}=\boldsymbol{\Theta}-\mathbf{\Theta}_{\mathrm{r}} \in \mathbb{R}^{3}$ and $\mathbf{E}_{\omega}=\boldsymbol{\omega}-\boldsymbol{\omega}_{\mathrm{r}} \in \mathbb{R}^{3}$, then the tracking error dynamics for the two subsystems can be written as

$$
\begin{aligned}
\dot{\mathbf{E}}_{\theta} & =\mathbf{R} \boldsymbol{\omega}+\boldsymbol{\Delta}_{f}-\dot{\Theta}_{\mathrm{r}} \\
\dot{\mathbf{E}}_{\omega} & =-\mathbf{I}^{-1} \boldsymbol{\Omega} \mathbf{I} \boldsymbol{\omega}+\mathbf{I}^{-1} \mathbf{M}+\boldsymbol{\Delta}_{d}-\dot{\boldsymbol{\omega}}_{\mathrm{r}}
\end{aligned}
$$

Note that a nominal version of Eq. (4) can be obtained by ignoring the effects caused by disturbances:

$$
\begin{aligned}
\dot{\tilde{\mathbf{E}}}_{\theta} & =\mathbf{R}(\tilde{\boldsymbol{\Theta}}) \tilde{\boldsymbol{\omega}}-\dot{\boldsymbol{\Theta}}_{\mathrm{r}} \\
\dot{\tilde{\mathbf{E}}}_{\omega} & =-\mathbf{I}^{-1} \boldsymbol{\Omega}(\tilde{\boldsymbol{\omega}}) \mathbf{I} \tilde{\boldsymbol{\omega}}+\mathbf{I}^{-1} \tilde{\mathbf{M}}-\dot{\boldsymbol{\omega}}_{\mathrm{r}}
\end{aligned}
$$

in which $\tilde{\boldsymbol{\Theta}}, \tilde{\boldsymbol{\omega}}$ and $\tilde{\mathbf{M}}$ denote the nominal angle, angular rate and control variables, respectively. $\tilde{\mathbf{E}}_{\omega}$ and $\tilde{\mathbf{E}}_{\theta}$ are the nominal tracking error vectors of the inner and outer loops, respectively.

For the second objective, the admissible sets of the angular and angular rate variables can be defined as $\mathbb{X}_{\Theta}=\{\boldsymbol{\Theta}$ : $\|\boldsymbol{\Theta}\| \leq \bar{\Theta}\}$ and $\mathbb{X}_{\omega}=\{\boldsymbol{\omega}:\|\boldsymbol{\omega}\| \leq \bar{\omega}\}$, where $\bar{\Theta}$ and $\bar{\omega}$ are two known positive constants. For the control moment $\mathbf{M}$, its admissible set is described as $\mathbb{U}_{M}=\{\mathbf{M}:\|\mathbf{M}\| \leq \bar{M}\}$, where $\bar{M}$ is a known positive constant. These types of system constraints must be frequently considered in practical applications, as a spacecraft can manoeuvre only within its mechanical limitations.

Remark 1. The proposed attitude tracking control scheme applies an inner-outer loop structure. To be more specific, the outer loop corresponds to the attitude angle subsystem (1a) and introduces a virtual control input $\boldsymbol{\omega}_{c}$, which is determined using the proposed outer-loop TRMPC algorithm (detailed in Sec III.A). Subsequently, this value is applied as the reference command (e.g., $\boldsymbol{\omega}_{\mathrm{r}}=\boldsymbol{\omega}_{c}$ ) and provided to the inner loop. Here, the angular rate subsystem (1b) is considered, and the actual control moment $\mathbf{M}$ is produced via the inner-loop TRMPC algorithm.

\section{Assumptions and Preliminaries}

To design the TRMPC algorithm, some assumptions are needed [6], [26].

Assumption 1. The Earth's rotation is ignored in the mission, and system variables $\Theta, \omega$ and $M$ are measurable.

Assumption 2. The attitude angle reference, the desired angular rate and their derivatives are considered to be smooth and bounded. That is, $\left\|\Theta_{\mathrm{r}}\right\| \leq \bar{\Theta}_{0},\left\|\dot{\Theta}_{\mathrm{r}}\right\| \leq \bar{\Theta}_{1},\left\|\boldsymbol{\omega}_{\mathrm{r}}\right\| \leq \bar{\omega}_{0}$, and $\left\|\dot{\omega}_{\mathrm{r}}\right\| \leq \bar{\omega}_{1}$.

Assumption 3. For the sideslip angle $\beta$, the case of $|\beta|=\frac{\pi}{2}$ is excluded from $\mathbb{X}_{\Theta}$ during the flight mission.

Assumption 4. The external disturbances are considered to be bounded. That is, $\left\|\boldsymbol{\Delta}_{f}\right\| \leq \eta_{1}$ and $\left\|\boldsymbol{\Delta}_{d}\right\| \leq \eta_{2}$.

These assumptions are adopted when deriving the main theoretical results of the proposed TRMPC. In addition, the following lemma reveals that with proper specification of $\Theta$, the 2-norm of $\mathbf{R}$ and $\mathbf{R}^{-1}$ has lower and upper bounds.

Lemma 1. If Assumption 3 is satisfied, the 2-norm of $\mathbf{R}(\mathbf{\Theta})$ and $\mathbf{R}^{-1}(\boldsymbol{\Theta})$ is bounded. That is, we have $\underline{r} \leq\|\mathbf{R}(\mathbf{\Theta})\| \leq \bar{r}$ and $\underline{r}^{\prime} \leq\left\|\mathbf{R}^{-1}(\boldsymbol{\Theta})\right\| \leq \bar{r}^{\prime}$.

Proof. The 2-norm of $\mathbf{R}(\boldsymbol{\Theta})$ can be computed via $\|\mathbf{R}(\boldsymbol{\Theta})\|=$ $\sqrt{\lambda_{\max }\left(\mathbf{R}^{T} \mathbf{R}\right)}$. Note that $\mathbf{R}^{T} \mathbf{R}$ and $\mathbf{R R}^{T}$ have same non-zero eigenvalues. By addressing $\left|\lambda \mathbf{I}-\mathbf{R R}^{T}\right|=0$, we can obtain $\lambda_{1}=1+\tan ^{2} \beta, \lambda_{2}=\lambda_{3}=1$. Hence, for any $\beta \in\left(-\frac{\pi}{2}, \frac{\pi}{2}\right)$, we have $\underline{r}=1$ and $\bar{r}=\sqrt{1+\tan ^{2} \beta}$. Moreover, from Eq. (2), we have $\mathbf{R}^{-1}$ in the form of

$$
\mathbf{R}^{-1}=\left[\begin{array}{ccc}
-\cos \alpha \sin \beta \cos \beta & \sin \alpha & -\cos \alpha \cos \beta \\
\cos ^{2} \beta & 0 & -\sin \beta \\
-\sin \alpha \sin \beta \cos \beta & -\cos \beta & -\sin \alpha \cos \beta
\end{array}\right]
$$

Its 2-norm is $\sqrt{\lambda_{\max }\left(\mathbf{R}^{-\mathbf{1}^{T}} \mathbf{R}^{-1}\right)}$. By solving $\mid \lambda \mathbf{I}-$ $\left(\mathbf{R}^{-1}\right)^{T} \mathbf{R}^{-1} \mid=0$, one can obtain $\lambda_{1}=\cos ^{2} \beta, \lambda_{2}=\lambda_{3}=1$. Hence, we have $\underline{r}^{\prime}=\bar{r}^{\prime}=1$, which completes the proof.

\section{Design of the TRMPC Algorithm}

This section presents a novel TRMPC design that is oriented from TMPC. Previous works on developing TMPC for linear system regulation problems can provide some guidelines [27], 
[28]. However, these tube-based designs cannot be directly applied to the considered tracking control problem due to the non-linear nature of the reference trajectory and the attitude dynamics. Hence, we devote efforts to extend the idea of tightening system constraints, constructing the terminal controller and invariant set, and designing the feedback law such that both robust constraint satisfaction and convergent tracking performance can be achieved for non-linear spacecraft attitude tracking systems. The designed TRMPC approach serves as the main controller to stabilize the actual tracking errors $\mathbf{E}_{\theta}$ and $\mathbf{E}_{\omega}$ toward the origin.

\section{A. Outer-Loop TRMPC Design}

In the outer attitude angle control loop, we can define the objective function at time instant $t_{k} \in\left\{t_{k}\right\}_{k \in \mathbb{N}}$ in the form of

$$
\begin{aligned}
J_{\theta}\left(\tilde{\mathbf{E}}_{\theta}\left(t_{k}\right), \tilde{\mathbf{U}}_{\theta}\left(t_{k}\right)\right)= & \int_{t_{k}}^{t_{k}+T} L_{\theta}\left(\tilde{\mathbf{E}}_{\theta}\left(\tau \mid t_{k}\right), \tilde{\mathbf{U}}_{\theta}\left(\tau \mid t_{k}\right)\right) d \tau \\
& +g_{\theta}\left(\tilde{\mathbf{E}}_{\theta}\left(t_{k}+T \mid t_{k}\right)\right)
\end{aligned}
$$

In Eq. (7), $L_{\theta}\left(\tilde{\mathbf{E}}_{\theta}\left(\tau \mid t_{k}\right), \tilde{\mathbf{U}}_{\theta}\left(\tau \mid t_{k}\right)\right)=\left\|\tilde{\mathbf{E}}_{\theta}\left(\tau \mid t_{k}\right)\right\|_{P}^{2}+$ $\left\|\tilde{\mathbf{U}}_{\theta}\left(\tau \mid t_{k}\right)\right\|_{Q}^{2}$ with $\tilde{\mathbf{U}}_{\theta}\left(\tau \mid t_{k}\right)=\mathbf{R}\left(\tilde{\boldsymbol{\Theta}}\left(\tau \mid t_{k}\right)\right) \tilde{\boldsymbol{\omega}}\left(\tau \mid t_{k}\right)-\dot{\boldsymbol{\Theta}}_{\mathrm{r}}$ and $g_{\theta}\left(\tilde{\mathbf{E}}_{\theta}\left(t_{k}+T \mid t_{k}\right)\right)=\left\|\tilde{\mathbf{E}}_{\theta}\left(t_{k}+T \mid t_{k}\right)\right\|_{R}^{2}$. The matrices $P, Q, R \in \mathbb{R}^{3 \times 3}$ are selected to be diagonal and positive definite. Their components (e.g., $P=\operatorname{diag}\left\{p_{i}\right\}_{i=1}^{3}, Q=$ $\operatorname{diag}\left\{q_{i}\right\}_{i=1}^{3}$ and $\left.R=\operatorname{diag}\left\{r_{i}\right\}_{i=1}^{3}\right)$ are chosen according to Lemma 2. $T$ denotes the predictive horizon.

As stated before, the entire control strategy involves two steps. First, an optimized control action, along with a central trajectory, is determined by addressing the following nominal system-based optimization model:

$$
\begin{array}{cl}
\min _{\tilde{\boldsymbol{\omega}}_{c}^{*}\left(\tau \mid t_{k}\right), \tilde{\boldsymbol{\Theta}}^{*}\left(\tau \mid t_{k}\right)} & J_{\theta}\left(\tilde{\mathbf{E}}_{\theta}\left(t_{k}\right), \tilde{\mathbf{U}}_{\theta}\left(t_{k}\right)\right) \\
\text { s.t. } & \forall \tau \in\left[t_{k}, t_{k}+T\right] \\
& \boldsymbol{\Theta}\left(t_{k}\right) \in \tilde{\boldsymbol{\Theta}}\left(t_{k} \mid t_{k}\right) \oplus \mathbb{O}_{\theta} \\
& \dot{\tilde{\boldsymbol{\Theta}}}\left(\tau \mid t_{k}\right)=\mathbf{R}(\tilde{\boldsymbol{\Theta}}) \tilde{\boldsymbol{\omega}}_{c} \\
& \dot{\tilde{\mathbf{E}}}_{\theta}\left(\tau \mid t_{k}\right)=\mathbf{R}(\tilde{\boldsymbol{\Theta}}) \tilde{\boldsymbol{\omega}}_{c}-\dot{\boldsymbol{\Theta}}_{\mathrm{r}} \\
& \tilde{\boldsymbol{\Theta}}\left(\tau \mid t_{k}\right) \in \mathbb{X}_{\theta}^{\text {tube }} \\
& \tilde{\boldsymbol{\omega}}_{c}\left(\tau \mid t_{k}\right) \in \mathbb{U}_{\omega}^{\text {tube }} \\
& \dot{\tilde{\mathbf{E}}}_{\theta}\left(t_{k}+T \mid t_{k}\right) \in \mathbf{\Omega}_{\theta}^{\text {tube }}
\end{array}
$$

in which

$$
\left\{\begin{array}{l}
\mathbb{X}_{\theta}^{\text {tube }}=\left\{\tilde{\boldsymbol{\Theta}}:\|\tilde{\boldsymbol{\Theta}}\| \leq \bar{\Theta}^{\text {tube }}\right\} \\
\bar{\Theta}^{\text {tube }}=\bar{\Theta}-\sqrt{3} \bar{k} \eta_{1}, \bar{k}=\max \left\{1 / k_{i}\right\}_{i=1}^{3} \\
\mathbb{U}_{\omega}^{\text {tube }}=\left\{\tilde{\boldsymbol{\omega}}_{c}:\left\|\tilde{\boldsymbol{\omega}}_{c}\right\| \leq \bar{\omega}_{c}^{\text {tube }}\right\} \\
\bar{\omega}_{c}^{\text {tube }}=\tilde{R} \bar{\omega}-\sqrt{3} \eta_{1}, \tilde{R}=\underline{r} / \bar{r} \\
\boldsymbol{\Omega}_{\theta}^{\text {tube }}=\left\{\tilde{\mathbf{E}}_{\theta}:\left\|\tilde{\mathbf{E}}_{\theta}\right\|_{R} \leq \epsilon_{\theta}\right\} \\
\epsilon_{\theta}=\sqrt{\lambda_{\min }(R)} \cdot \min \left\{\frac{\bar{\omega}_{c}^{\text {tube }}-\bar{r}^{\prime} \bar{\Theta}_{1}}{\left\|\bar{r}^{\prime} \tilde{\mathbf{K}}\right\|}, \bar{\Theta}-\bar{\Theta}_{0}-\sqrt{3} \bar{k} \eta_{1}\right\}
\end{array}\right.
$$

In Eq. (8) and Eq. (9), $\mathbb{X}_{\theta}^{\text {tube }}$ and $\mathbb{U}_{\omega}^{\text {tube }}$ are, respectively, the tightened state and input constraints. Two gain matrices (e.g., $\mathbf{K}$ and $\tilde{\mathbf{K}}$ ), along with an initial state set $\mathbb{O}_{\theta}$, are introduced. Their values can be determined offline by following the results shown in the next two lemmas. Specifically, in Lemma 2, some hints regarding how to construct a terminal controller $\tilde{\boldsymbol{\omega}}_{c}^{f}$ and the corresponding terminal invariant set $\Omega_{\theta}^{\text {tube }}$ are provided. Before presenting Lemma 2, we introduce Definition 1:
Definition 1. For the error system (5a), $\tilde{\boldsymbol{\omega}}_{c}^{f}$ and $\Omega_{\theta}^{\text {tube }}$ are the terminal controller and the terminal invariant set if the following conditions hold true for any $\tau \in\left[t_{k}+T, t_{k+1}+T\right]$ :

$$
\left\{\begin{array}{l}
\tilde{\boldsymbol{\Theta}}\left(\tau \mid t_{k}\right) \in \mathbb{X}_{\theta}^{\text {tube }} \\
\tilde{\boldsymbol{\omega}}_{c}^{f}\left(\tau \mid t_{k}\right) \in \mathbb{U}_{\omega}^{\text {tube }} \\
\dot{g}_{\theta}\left(\tilde{\mathbf{E}}_{\theta}\left(\tau \mid t_{k}\right)\right)+L_{\theta}\left(\tilde{\mathbf{E}}_{\theta}\left(\tau \mid t_{k}\right), \tilde{\mathbf{U}}_{\theta}\left(\tau \mid t_{k}\right)\right) \leq 0
\end{array}\right.
$$

Lemma 2. $\Omega_{\theta}^{\text {tube }}$, defined by Eqs. (9e)-(9f), is a terminal invariant set for system (5a) resulting from the terminal controller:

$$
\tilde{\boldsymbol{\omega}}_{c}^{f}=\mathbf{R}^{-1}(\tilde{\mathbf{\Theta}}) \tilde{\mathbf{K}} \tilde{\mathbf{E}}_{\theta}+\mathbf{R}^{-1}(\tilde{\mathbf{\Theta}}) \dot{\boldsymbol{\Theta}}_{r}
$$

where $\tilde{\mathbf{K}}=\operatorname{diag}\left\{\tilde{k}_{i}\right\}_{i=1}^{3}$. In addition, the parameters satisfy $p_{i} q_{i}<\frac{r_{i}^{2}}{4}$ and $\tilde{k}_{i} \in\left(\frac{r_{i}-\sqrt{r_{i}^{2}-4 p_{i} q_{i}}}{2 q_{i}}, \frac{r_{i}^{2}+\sqrt{r_{i}^{2}-4 p_{i} q_{i}}}{2 q_{i}}\right)$.

Proof. First, attention is paid to the nominal state variable $\tilde{\boldsymbol{\Theta}}$ when it enters $\boldsymbol{\Omega}_{\theta}^{\text {tube }}$. From (5a), we have $\tilde{\boldsymbol{\Theta}}=\tilde{\mathbf{E}}_{\theta}+\boldsymbol{\Theta}_{\mathrm{r}}$ and

$$
\|\tilde{\boldsymbol{\Theta}}\|=\frac{\left\|\tilde{\mathbf{E}}_{\theta}\right\|_{R}}{\sqrt{\lambda_{\min }(R)}}+\left\|\boldsymbol{\Theta}_{\mathrm{r}}\right\|
$$

Since $\left\|\tilde{\mathbf{E}}_{\theta}\right\|_{R} \leq \epsilon_{\theta}$ and Eq. (9f) hold, we have

$$
\begin{aligned}
\|\tilde{\boldsymbol{\Theta}}\| & \leq \frac{\epsilon_{\theta}}{\sqrt{\lambda_{\min }(R)}}+\left\|\boldsymbol{\Theta}_{\mathrm{r}}\right\| \\
& \leq \bar{\Theta}-\sqrt{3} \bar{k} \eta_{1}=\bar{\Theta}^{\text {tube }}
\end{aligned}
$$

Moreover, based on the expression of $\tilde{\boldsymbol{\omega}}_{c}^{f}$ given by (11) and Lemma 1 , it can be obtained that

$$
\begin{aligned}
\left\|\tilde{\boldsymbol{\omega}}_{c}^{f}\right\| & \leq\left\|\mathbf{R}^{-1}(\tilde{\boldsymbol{\Theta}})\right\|\|\tilde{\mathbf{K}}\| \frac{\left\|\tilde{\mathbf{E}}_{\theta}\right\|_{R}}{\sqrt{\lambda_{\min }(R)}}+\left\|\mathbf{R}^{-1}(\tilde{\boldsymbol{\Theta}})\right\|\left\|\dot{\boldsymbol{\Theta}}_{\mathrm{r}}\right\| \\
& \leq \bar{r}^{\prime}\|\tilde{\mathbf{K}}\| \frac{\epsilon_{\theta}}{\sqrt{\lambda_{\min }(R)}}+\bar{r}^{\prime} \bar{\Theta}_{1} \leq \bar{\omega}^{\text {tube }}
\end{aligned}
$$

Next, if we apply $\tilde{\boldsymbol{\omega}}_{c}^{f}$ over $\tau \in\left[t_{k}+T, t_{k+1}+T\right)$, the derivative of $J_{\theta}$ becomes

$$
\begin{aligned}
\dot{g}_{\theta} & \left(\tilde{\mathbf{E}}_{\theta}\left(\tau \mid t_{k}\right)\right)+L_{\theta}\left(\tilde{\mathbf{E}}_{\theta}\left(\tau \mid t_{k}\right),-\tilde{\mathbf{K}} \tilde{\mathbf{E}}_{\theta}\left(\tau \mid t_{k}\right)\right) \\
= & \left\|\tilde{\mathbf{K}} \tilde{\mathbf{E}}_{\theta}\left(\tau \mid t_{k}\right)\right\|_{Q}^{2}-\tilde{\mathbf{K}}\left\|\tilde{\mathbf{E}}{ }_{\theta}\left(\tau \mid t_{k}\right)\right\|_{R}^{2}+\left\|\tilde{\mathbf{E}}_{\theta}\left(\tau \mid t_{k}\right)\right\|_{R}^{2} \\
\leq & \|\tilde{\mathbf{K}}\|^{2}\left\|\tilde{\mathbf{E}}_{\theta}\left(\tau \mid t_{k}\right)\right\|_{Q}^{2}-\tilde{\mathbf{K}}\left\|\tilde{\mathbf{E}} \theta\left(\tau \mid t_{k}\right)\right\|_{R}^{2} \\
& +\left\|\tilde{\mathbf{E}_{\theta}}\left(\tau \mid t_{k}\right)\right\|_{P}^{2}
\end{aligned}
$$

Since $\tilde{k}_{i} \in\left(\frac{r_{i}^{2}-\sqrt{r_{i}^{2}-4 p_{i} q_{i}}}{2 q_{i}}, \frac{r_{i}^{2}+\sqrt{r_{i}^{2}-4 p_{i} q_{i}}}{2 q_{i}}\right)$, it is obvious that

$$
-r_{i} \tilde{k}_{i}+p_{i}+q_{i} \tilde{k}_{i}^{2}<0
$$

Substituting Eq. (16) into Eq. (15) yields the stability condition $\dot{g}_{\theta}+L_{\theta}<0$. Until now, we have verified all conditions stated in Definition 1, indicating that $\tilde{\boldsymbol{\omega}}_{c}^{f}$ and $\boldsymbol{\Omega}_{\theta}^{\text {tube }}$ are the terminal controller and the corresponding invariant set for system (5a).

Once the optimized control law and the resulting nominal trajectory are obtained (denoted as $\tilde{\boldsymbol{\omega}}_{c}{ }^{*}\left(\tau \mid t_{k}\right)$ and $\tilde{\boldsymbol{\Theta}}^{*}\left(\tau \mid t_{k}\right)$, respectively, for $\tau \in\left[t_{k}, t_{k}+T\right]$ ), we further design a robust control law such that it can be applied to the actual angle system. Due to the existence of disturbances, applying $\tilde{\boldsymbol{\omega}}_{c}{ }^{*}$ to the actual system over $\tau \in\left[t_{k}, t_{k+1}\right)$ may result in a deviation between the actual angular profile and the optimized one. That 
is, $O_{\theta}(\tau)=\boldsymbol{\Theta}(\tau)-\tilde{\boldsymbol{\Theta}}^{*}\left(\tau \mid t_{k}\right)$. Differentiating $O_{\theta}(\tau)$, we have

$$
\dot{O}_{\theta}(\tau)=\mathbf{R}(\boldsymbol{\Theta}) \boldsymbol{\omega}_{c}(\tau)-\mathbf{R}\left(\tilde{\boldsymbol{\Theta}}^{*}\right) \tilde{\boldsymbol{\omega}}_{c}^{*}\left(\tau \mid t_{k}\right)+\boldsymbol{\Delta}_{f}
$$

Then, we can design the robust control law in the form of

$$
\boldsymbol{\omega}_{c}(\tau)=\mathbf{R}^{-1}(\boldsymbol{\Theta})\left[\mathbf{R}\left(\tilde{\boldsymbol{\Theta}}^{*}\right) \tilde{\boldsymbol{\omega}}_{c}^{*}\left(\tau \mid t_{k}\right)-\mathbf{K} O_{\theta}(\tau)\right]
$$

where $\mathbf{K}=\operatorname{diag}\left\{k_{i}\right\}_{i=1}^{3}, k_{i}>0$. The next lemma reveals that by applying the robust control law (18), the actual angular trajectory can stay within the tube region centred around the nominal solution while simultaneously satisfying the actual state and input constraints.

Lemma 3. If $\boldsymbol{\omega}_{c}(\tau)$ is applied to steer the actual attitude angle system (la) over $\tau \in\left[t_{k}, t_{k+1}\right)$ and $\boldsymbol{\Delta}_{f}$ is upper bounded by $\eta_{1}$, we have:

1) The system state and input constraints can be satisfied. That is, $\boldsymbol{\Theta}(\tau) \in \mathbb{X}_{\Theta}$ and $\boldsymbol{\omega}_{c}(\tau) \in \mathbb{X}_{\omega}$

2) The actual attitude angle trajectory can stay within $\Theta(\tau) \in$ $\tilde{\boldsymbol{\Theta}}^{*}\left(\tau \mid t_{k}\right) \oplus \mathbb{O}_{\theta}$, where $\mathbb{O}_{\theta}=\left\{O_{\theta}(\tau):\left|O_{\theta}\right| \leq\right.$ $\left.\left[-\eta_{1} / k_{1},-\eta_{1} / k_{2},-\eta_{1} / k_{3}\right]^{T}\right\}$.

Proof. For 1), by substituting $\boldsymbol{\omega}_{c}(\tau)$ into $\dot{O}_{\theta}(\tau)$, we have

$$
\dot{O}_{\theta}(\tau)=\boldsymbol{\Delta}_{f}(\tau)+\mathbf{K} O_{\theta}(t), O_{\theta}(0)=0
$$

The solution of (19) can be written as

$$
O_{\theta}(\tau)=e^{\mathbf{K} \tau} O_{\theta}(0)+\int_{0}^{\tau} e^{\mathbf{K}(\tau-t)} \boldsymbol{\Delta}_{f}(t) d t
$$

Since $\left\|\Delta_{f}\right\| \leq \eta_{1}$, one can obtain

$$
\left\|O_{\theta}(\tau)\right\| \leq \eta_{1}\left\|\left[1 / k_{1}, 1 / k_{2}, 1 / k_{3}\right]^{T}\right\| \leq \sqrt{3} \bar{k} \eta_{1}
$$

Based on the definition of $O_{\theta}(\tau)$, it holds that

$$
\begin{aligned}
\|\Theta(\tau)\| & =\left\|\tilde{\boldsymbol{\Theta}}^{*}\left(\tau \mid t_{k}\right)+O_{\theta}(\tau)\right\| \\
& \leq\left\|\tilde{\boldsymbol{\Theta}}^{*}\left(\tau \mid t_{k}\right)\right\|+\left\|O_{\theta}(\tau)\right\| \\
& \leq \bar{\Theta}^{\text {tube }}+\sqrt{3} \bar{k} \eta_{1}=\bar{\Theta}
\end{aligned}
$$

which indicates that $\Theta \in \mathbb{X}_{\Theta}$. Moreover, if we define

$$
\left\{\begin{array}{l}
\hat{\boldsymbol{\omega}}_{c}=\mathbf{R}(\boldsymbol{\Theta}) \boldsymbol{\omega}_{c} \\
\hat{\boldsymbol{\omega}}_{c}^{*}=\mathbf{R}\left(\tilde{\boldsymbol{\Theta}}^{*}\right) \tilde{\boldsymbol{\omega}}_{c}^{*}
\end{array}\right.
$$

then it is obvious that

$$
\hat{\boldsymbol{\omega}}_{c}=\hat{\boldsymbol{\omega}}_{c}^{*}+\mathbf{K} O_{\theta}
$$

We further define two sets:

$$
\left\{\begin{array}{l}
\hat{\mathbb{U}}_{\omega}=\left\{\hat{\boldsymbol{\omega}}_{c}:\left\|\hat{\boldsymbol{\omega}}_{c}\right\| \leq \underline{r} \bar{\omega}\right\} \\
\hat{\mathbb{U}}_{\omega}^{\text {tube }}=\left\{\hat{\boldsymbol{\omega}}_{c}^{*}:\left\|\hat{\boldsymbol{\omega}}_{c}^{*}\right\| \leq \bar{r} \bar{\omega}_{c}^{\text {tube }}\right\}
\end{array}\right.
$$

From (21), one can write

$$
\begin{aligned}
\mathbb{O}_{\theta} & =\left\{O_{\theta}(\tau):\left\|O_{\theta}(\tau)\right\| \leq \eta_{1}\left[1 / k_{1}, 1 / k_{3}, 1 / k_{3}\right]^{T}\right\} \\
& \subset\left\{O_{\theta}(\tau):\left\|O_{\theta}(\tau)\right\| \leq \sqrt{3} \bar{k} \eta_{1}\right\}
\end{aligned}
$$

After investigating $\mathbb{O}_{\theta}$, $\hat{\mathbb{U}}_{\omega}$ and $\hat{\mathbb{U}}_{\omega}^{\text {tube }}$, it follows that

$$
\hat{\mathbb{U}}_{\omega}^{\text {tube }} \oplus \mathbf{K} \mathbb{O}_{\theta} \in \hat{\mathbb{U}}_{\omega}
$$

Thus, if $\tilde{\boldsymbol{\omega}}_{c}^{*} \in \hat{\mathbb{U}}_{\omega}^{\text {tube }}$, based on (23)-(27), we have $\hat{\boldsymbol{\omega}}_{c}^{*} \in \hat{\mathbb{U}}_{\omega}^{\text {tube }}$ and $\hat{\boldsymbol{\omega}}_{c} \in \hat{\mathbb{U}}_{\omega}$. Subsequently, based on (23a) and (9d), one can conclude that $\boldsymbol{\omega}_{c} \in \mathbb{X}_{\omega}$.

For 2), since $\boldsymbol{\Theta}(\tau)=\tilde{\boldsymbol{\Theta}}^{*}\left(\tau \mid t_{k}\right)+O_{\theta}(\tau)$, it is true that $\boldsymbol{\Theta}(\tau) \in \tilde{\boldsymbol{\Theta}}^{*}\left(\tau \mid t_{k}\right) \oplus \mathbb{O}_{\theta}$, indicating that the system state will stay within a tube region centred around the nominal solution.

\section{B. Inner-Loop TRMPC Design}

Results from the outer loop are applied as the reference command $\omega_{\mathrm{r}}$ to design the inner-loop tracking controller. Similar to that of the outer loop, the inner-loop objective function can be given by

$$
\begin{aligned}
J_{\omega}\left(\tilde{\mathbf{E}}_{\omega}\left(t_{k}\right), \tilde{\mathbf{U}}_{\omega}\left(t_{k}\right)\right)= & \int_{t_{k}}^{t_{k}+T} L_{\omega}\left(\tilde{\mathbf{E}}_{\omega}\left(\tau \mid t_{k}\right), \tilde{\mathbf{U}}_{\omega}\left(\tau \mid t_{k}\right)\right) d \tau \\
& +g_{\omega}\left(\tilde{\mathbf{E}}_{\omega}\left(t_{k}+T \mid t_{k}\right)\right)
\end{aligned}
$$

in which $L_{\omega}\left(\tilde{\mathbf{E}}_{\omega}\left(\tau \mid t_{k}\right), \tilde{\mathbf{U}}_{\omega}\left(\tau \mid t_{k}\right)\right)=\left\|\tilde{\mathbf{E}}_{\omega}\left(\tau \mid t_{k}\right)\right\|_{P}^{2}+$ $\left\|\tilde{\mathbf{U}}_{\omega}\left(\tau \mid t_{k}\right)\right\|_{Q}^{2}$ with $\tilde{\mathbf{U}}_{\omega}\left(\tau \mid t_{k}\right)=-\mathbf{I}^{-1} \boldsymbol{\Omega}(\tilde{\boldsymbol{\omega}}) \mathbf{I} \tilde{\boldsymbol{\omega}}\left(\tau \mid t_{k}\right)-\boldsymbol{\omega}_{\mathrm{r}}+$ $\mathbf{I}^{-1} \tilde{\mathbf{M}}\left(\tau \mid t_{k}\right)$ and $g_{\omega}\left(\tilde{\mathbf{E}}_{\omega}\left(t_{k}+T \mid t_{k}\right)\right)=\left\|\tilde{\mathbf{E}}_{\omega}\left(t_{k}+T \mid t_{k}\right)\right\|_{R}^{2}$. Again, the components of the matrices $P, Q, R$ are chosen according to Lemma 4.

Similar to Eq. (8), the optimization model in the inner loop can be formulated as:

$$
\begin{array}{cl}
\min _{\tilde{\boldsymbol{\omega}}^{*}\left(\tau \mid t_{k}\right), \tilde{\mathbf{M}}^{*}\left(\tau \mid t_{k}\right)} & J_{\omega}\left(\tilde{\mathbf{E}}_{\omega}\left(t_{k}\right), \tilde{\mathbf{U}}_{\omega}\left(t_{k}\right)\right) \\
\text { s.t. } & \forall \tau \in\left[t_{k}, t_{k}+T\right] \\
& \boldsymbol{\omega}\left(t_{k}\right) \in \tilde{\boldsymbol{\omega}}\left(t_{k} \mid t_{k}\right) \oplus \mathbb{O}_{\omega} \\
& \dot{\tilde{\boldsymbol{\omega}}}\left(\tau \mid t_{k}\right)=-\mathbf{I}^{-1} \boldsymbol{\Omega}(\tilde{\boldsymbol{\omega}}) \mathbf{I} \tilde{\boldsymbol{\omega}}+\mathbf{I}^{-1} \tilde{\mathbf{M}} \\
& \dot{\tilde{\mathbf{E}}} \omega\left(\tau \mid t_{k}\right)=-\mathbf{I}^{-1} \boldsymbol{\Omega}(\tilde{\boldsymbol{\omega}}) \mathbf{I} \tilde{\boldsymbol{\omega}}+\mathbf{I}^{-1} \tilde{\mathbf{M}}-\dot{\boldsymbol{\omega}}_{\mathrm{r}} \\
& \tilde{\boldsymbol{\omega}}\left(\tau \mid t_{k}\right) \in \mathbb{X}_{\omega}^{\text {tube }} \\
& \tilde{\mathbf{M}}\left(\tau \mid t_{k}\right) \in \mathbb{U}_{M}^{\text {tube }} \\
& \dot{\tilde{\mathbf{E}}}_{\omega}\left(t_{k}+T \mid t_{k}\right) \in \mathbf{\Omega}_{\omega}^{\text {tube }}
\end{array}
$$

in which

$$
\left\{\begin{array}{l}
\mathbb{X}_{\omega}^{\text {tube }}=\left\{\tilde{\boldsymbol{\omega}}:\|\tilde{\boldsymbol{\omega}}\| \leq \bar{\omega}^{\text {tube }}\right\} \\
\bar{\omega}^{\text {tube }}=\bar{\omega}-\sqrt{3} \bar{k} \eta_{2}, \bar{k}=\max \left\{1 / k_{i}\right\}_{i=1}^{3} \\
\mathbb{U}_{M}^{\text {tube }}=\left\{\tilde{\mathbf{M}}:\|\tilde{\mathbf{M}}\| \leq \bar{M}^{\text {tube }}\right\} \\
\bar{M}^{\text {tube }}=\bar{M}-\bar{c}+\bar{d}-\sqrt{3}\|\mathbf{I}\| \eta_{2} \\
\boldsymbol{\Omega}_{\omega}^{\text {tube }}=\left\{\tilde{\mathbf{E}}_{\omega}:\left\|\tilde{\mathbf{E}}_{\omega}\right\|_{R} \leq \epsilon_{\omega}\right\} \\
\epsilon_{\omega}=\sqrt{\lambda_{\min }(R)} \cdot \min \left\{\frac{\bar{M}^{\text {tube }}-\bar{d}-\bar{\omega}_{1}\|\mathbf{I}\|}{\|\tilde{\mathbf{K}}\|\|\mathbf{I}\|}, \bar{\omega}^{\text {tube }}-\bar{\omega}_{0}\right\}
\end{array}\right.
$$

To construct the terminal controller $\tilde{\mathbf{M}}^{f}$ and the terminal invariant set $\Omega_{\omega}^{\text {tube }}$, the following lemma is derived.

Lemma 4. $\Omega_{\omega}^{\text {tube }}$, defined by Eqs. (30e)-(30f), is a terminal invariant set for the system $(5 b)$ resulting from the terminal controller:

$$
\tilde{\mathbf{M}}^{f}=\Omega(\tilde{\omega}) \mathbf{I} \tilde{\omega}+\mathbf{I} \dot{\boldsymbol{\omega}}_{r}+\mathbf{I} \tilde{\mathbf{K}} \tilde{\mathbf{E}}_{\omega}
$$

where $\tilde{\mathbf{K}}=\operatorname{diag}\left\{\tilde{k}_{i}\right\}_{i=1}^{3}$. In addition, the parameters satisfy $p_{i} q_{i}<\frac{r_{i}^{2}}{4}$ and $k_{i} \in\left(\frac{r_{i}-\sqrt{r_{i}^{2}-4 p_{i} q_{i}}}{2 q_{i}}, \frac{r_{i}^{2}+\sqrt{r_{i}^{2}-4 p_{i} q_{i}}}{2 q_{i}}\right)$.

Proof. Based on the expression of $\tilde{\mathbf{M}}^{f}$ in (31), one can write

$$
\left\|\tilde{\mathbf{M}}^{f}\right\| \leq\|\Omega(\tilde{\omega}) \mathbf{I} \tilde{\omega}\|+\|\mathbf{I}\|\left\|\dot{\boldsymbol{\omega}}_{\mathrm{r}}\right\|+\|\mathbf{I}\|\|\tilde{\mathbf{K}}\|\left\|\tilde{\mathbf{E}}_{\omega}\right\|
$$

Note that according to (3), the term $\Omega(\omega) \mathbf{I} \omega=F(\omega)$ can be written as

$$
F(\omega)=\left[\begin{array}{c}
F_{1}(\omega) \\
F_{2}(\omega) \\
F_{3}(\omega)
\end{array}\right]=\left[\begin{array}{c}
-p q I_{x z}-q \nu I_{y y}+q \nu I_{z z} \\
p \nu I_{x x}-p^{2} I_{x z}-\nu^{2} I_{x z}-p \nu I_{z z} \\
-p q I_{x x}+p q I_{y y}+q \nu I_{x z}
\end{array}\right]
$$


Define

$$
\begin{aligned}
& \bar{c}=\arg \max _{\omega \in\{\omega:\|\omega\| \leq \bar{\omega}\}} F(\omega) \\
& \bar{d}=\arg \max _{\tilde{\omega} \in \mathbb{X}_{\omega}^{\text {tube }}} F(\omega)
\end{aligned}
$$

Eq. (32) can be further written as

$$
\begin{aligned}
\left\|\tilde{\mathbf{M}}^{f}\right\| & \leq \bar{d}+\bar{\omega}_{1}\|\mathbf{I}\|+\|\mathbf{I}\|\|\tilde{\mathbf{K}}\|\left\|\tilde{\mathbf{E}}_{\omega}\right\| \\
& \leq \bar{d}+\bar{\omega}_{1}\|\mathbf{I}\|+\|\mathbf{I}\|\|\tilde{\mathbf{K}}\| \frac{\left\|\tilde{\mathbf{E}}_{\omega}\right\|_{R}}{\sqrt{\lambda_{\min }(R)}} \leq \bar{M}^{\text {tube }}
\end{aligned}
$$

which implies $\tilde{\mathbf{M}}^{f} \in \mathbb{U}_{M}^{\text {tube }}$. The rest of the proof follows a similar procedure to that of Lemma 2 and thus is omitted here.

Similar to the case of the outer loop, the deviation $O_{\omega}(\tau)$ between the actual and nominal angular rate trajectory when applying $\tilde{\mathbf{M}}\left(\tau \mid t_{k}\right)$ to system (1b) over $\left[t_{k}, t_{k+1}\right)$ is analysed. More precisely, we have

$$
\begin{aligned}
\dot{O}_{\omega}(\tau)= & -\mathbf{I}^{-1} \boldsymbol{\Omega}(\boldsymbol{\omega}) \mathbf{I} \boldsymbol{\omega}(\tau)+\mathbf{I}^{-1} \mathbf{M}(\tau) \\
& +\mathbf{I}^{-1} \boldsymbol{\Omega}(\tilde{\boldsymbol{\omega}}) \mathbf{I} \tilde{\boldsymbol{\omega}}^{*}\left(\tau \mid t_{k}\right)-\mathbf{I}^{-1} \tilde{\mathbf{M}}^{*}\left(\tau \mid t_{k}\right)+\boldsymbol{\Delta}_{d}
\end{aligned}
$$

Then, a compound angular rate control law can be given by:

$$
\mathbf{M}(\tau)=\boldsymbol{\Omega}(\boldsymbol{\omega}) \mathbf{I} \boldsymbol{\omega}(\tau)-\boldsymbol{\Omega}\left(\tilde{\boldsymbol{\omega}}^{*}\right) \mathbf{I} \tilde{\boldsymbol{\omega}}^{*}\left(\tau \mid t_{k}\right)+\tilde{\mathbf{M}}^{*}\left(\tau \mid t_{k}\right)-\mathbf{I K} O_{\omega}(\tau)
$$

The robust control law (36) is then applied to the actual angular rate system, and Lemma 5 provides some insights regarding the actual angular rate trajectory and the robust constraint satisfaction.

Lemma 5. If $\mathbf{M}(\tau)$ is applied to steer the actual angular rate system (1b) over $\tau \in\left[t_{k}, t_{k+1}\right)$ and $\boldsymbol{\Delta}_{d}$ is upper bounded by $\eta_{2}$, we have the following:

1) The angular rate and control moment constraints can be satisfied. That is, $\boldsymbol{\omega}(\tau) \in \mathbb{X}_{\omega}$ and $\mathbf{M}(\tau) \in \mathbb{U}_{M}$;

2) The actual angular rate trajectory can stay within $\boldsymbol{\omega}(\tau) \in$ $\tilde{\boldsymbol{\omega}}^{*}\left(\tau \mid t_{k}\right) \oplus \mathbb{O}_{\omega}$. Here, $\mathbb{O}_{\omega}=\left\{O_{\theta}(\tau):\left|O_{\theta}\right| \leq\right.$ $\left.\left[-\eta_{2} / k_{1},-\eta_{2} / k_{2},-\eta_{2} / k_{3}\right]^{T}\right\}$.

Proof. The proof follows a similar procedure to that of Lemma 3 and thus is omitted here.

\section{Overall Algorithm Framework}

A schematic diagram of the spacecraft attitude system under control is visualized in Fig. 1. To detail the implementation of

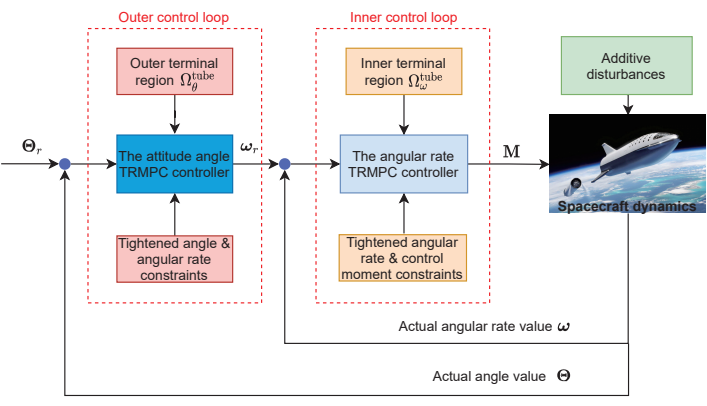

Fig. 1: Schematic diagram of the entire system

the TRMPC scheme, the inner angular rate control loop shown in Fig. 1 is extracted and expanded in Fig. 2. The corresponding implementation steps are summarised in Algorithm 1.

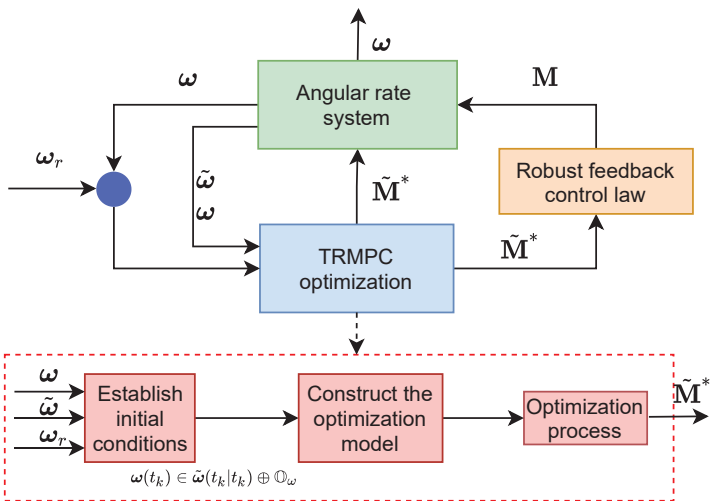

Fig. 2: Implementation of the TRMPC scheme

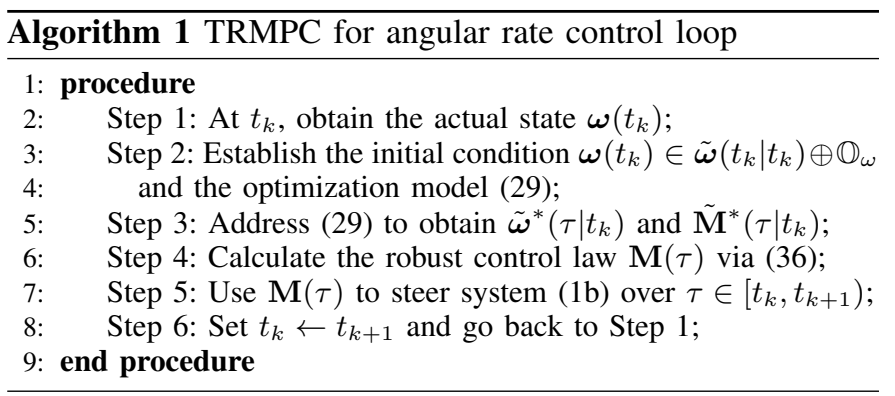

Remark 2. Note that the proposed dual-loop TRMPC tracking controller is robust against external disturbances, which is mainly achieved by tightening the system variable constraints for the nominal optimization model, thereby taking the effect of disturbances into consideration explicitly. Then, as demonstrated in Algorithm 1, the optimized solution is used to construct the robust feedback control law. More specifically, the robust expressions of the proposed inner and outer TRMPC can be abbreviated as $\boldsymbol{\omega}_{c}(\tau)=\kappa_{\omega}\left(\tilde{\boldsymbol{\Theta}}^{*}, \tilde{\boldsymbol{\omega}}_{c}^{*}, \boldsymbol{\Theta}\right)$ and $\mathbf{M}(\tau)=\kappa_{M}\left(\tilde{\boldsymbol{\omega}}^{*}, \tilde{\mathbf{M}}^{*}, \boldsymbol{\omega}\right)$. Here, $\left(\tilde{\boldsymbol{\Theta}}^{*}, \tilde{\boldsymbol{\omega}}_{c}^{*}\right)$ and $\left(\tilde{\boldsymbol{\omega}}^{*}, \tilde{\mathbf{M}}^{*}\right)$ are the solutions to optimization models (8) and (29), respectively. As discussed in Lemma 3 and Lemma 5, robust constraint satisfaction can be achieved if $\boldsymbol{\omega}_{c}(\tau)$ and $\mathbf{M}(\tau)$ are applied to control the systems (1a) and (1b). Moreover, the proposed compound control law can steer the actual state trajectory such that it stays within a tube region, which is regulated by the optimized state trajectory, the feedback gain matrix $\mathbf{K}$ and the upper bounds of the external disturbances $\left(\eta_{1}\right.$ or $\left.\eta_{2}\right)$.

Remark 3. Different from other related works [23], [24], where the robust feedback law was obtained by addressing another MPC to attenuate the effect of additive disturbances, the feedback laws given by (18) and (36) are determined offline via feedback linearization of (17) and (35), respectively. This means that at time instant $t_{k}$, only a nominal system-based open-loop optimization problem has to be solved in real time. Although this strategy might bring difficulties in designing tightened state/control constraints, one key advantage is that no additional optimization process is required, thereby alleviating the online computational burden significantly. Moreover, it is likely to facilitate practical implementations of the algorithm for various engineering problems. 


\section{ANALYSIS OF FEASIBILITY AND STABILITY}

\section{A. Recursive Feasibility}

In this section, the main theoretical properties of the proposed TRMPC are analysed. To be more specific, Theorem 1 is established to show the recursive feasibility of applying the proposed TRMPC control scheme.

Theorem 1. Given the outer and inner tracking error systems in the form of (4a) and (4b), if a feasible control solution for optimization problems (8) and (29) at time point $t_{k}$ can be found, then the optimization problems are recursively feasible for all $t_{k+1}>t_{k}$.

Proof. First, attention is paid to the outer tracking system (4a) and optimization problem (8). Assume that an optimal control solution $\tilde{\boldsymbol{\omega}}_{c}^{*}\left(\tau \mid t_{k}\right)$ is successfully found by solving (8). Then, the robust control law (18) is calculated and applied to the actual attitude angle system over $\left[t_{k}, t_{k+1}\right]$. From Lemma 3 , we have $\boldsymbol{\Theta}\left(t_{k+1}\right) \in \tilde{\boldsymbol{\Theta}}^{*}\left(t_{k+1} \mid t_{k}\right) \oplus \mathbb{O}_{\theta}$, indicating that $\tilde{\boldsymbol{\Theta}}\left(t_{k+1}\right)=\tilde{\boldsymbol{\Theta}}^{*}\left(t_{k+1} \mid t_{k}\right)$ is a feasible nominal attitude angle value for optimization problem (8) at time instant $t_{k+1}$. With this initial specification, a candidate control sequence for problem (8) at $t_{k+1}$ can be designed as

$$
\tilde{\boldsymbol{\omega}}_{c}\left(\tau \mid t_{k+1}\right)=\left\{\begin{array}{lll}
\tilde{\boldsymbol{\omega}}_{c}^{*}\left(\tau \mid t_{k}\right) & \text { if } & \tau \in\left[t_{k+1}, t_{k}+T\right) \\
\tilde{\boldsymbol{\omega}}_{c}^{f}\left(\tau \mid t_{k}\right) & \text { if } & \tau \in\left[t_{k}+T, t_{k+1}+T\right)
\end{array}\right.
$$

Note that this candidate control sequence consists of two parts: the tail of $\tilde{\boldsymbol{\omega}}_{c}^{*}$ and the terminal control law given by (10). To illustrate that $\tilde{\boldsymbol{\omega}}_{c}\left(\tau \mid t_{k+1}\right)$ is a feasible solution at $t_{k+1}$, Lemma 2 is recalled. It is obvious that $\tilde{\boldsymbol{\omega}}_{c}\left(\tau \mid t_{k+1}\right)$ is able to steer $\tilde{\mathbf{E}}_{\theta}\left(\tau \mid t_{k+1}\right)$ into $\boldsymbol{\Omega}_{\theta}^{\text {tube }}$ for $\tau \in\left[t_{k+1}, t_{k}+T\right)$ without violating the tightened state and control constraints. Since $\boldsymbol{\Omega}_{\theta}^{\text {tube }}$ is a terminal invariant set, we have $\tilde{\mathbf{E}}_{\theta}\left(\tau \mid t_{k+1}\right) \in \boldsymbol{\Omega}_{\theta}^{\text {tube }}$ under the terminal control $\tilde{\boldsymbol{\omega}}_{c}\left(\tau \mid t_{k+1}\right)$ over $\left[t_{k}+T, t_{k+1}+T\right)$. This also implies that $\tilde{\boldsymbol{\Theta}}\left(\tau \mid t_{k+1}\right) \in \mathbb{X}_{\theta}^{\text {tube }}$ and $\tilde{\boldsymbol{\omega}}_{c}\left(\tau \mid t_{k+1}\right) \in \mathbb{U}_{\omega}^{\text {tube }}$. Hence, $\tilde{\boldsymbol{\omega}}_{c}\left(\tau \mid t_{k+1}\right)$ is a feasible solution for (8) at $t_{k+1}$, and by induction, the proof can be completed.

For the inner tracking system (4b) and optimization problem (29), the proof follows the same procedure as that of the outer loop. Thus, we omit this part.

\section{B. Control Stability}

The next theorem (e.g., Theorem 2) reveals that when using the proposed control scheme, the ISS of the tracking error system can be guaranteed. This further ensures that the timevarying attitude angle profiles can be successfully tracked.

Theorem 2. If the optimization process of the dual-loop TRMPC-based attitude control algorithm is feasible at $t_{k}$, then the outer and inner tracking error systems have ISS.

Proof. The proof is shown in the Appendix.

\section{Performance Evaluation}

\section{A. Parameter Assignment}

To validate the effectiveness and performance of the proposed dual-loop TRMPC design, a number of experimental studies and analyses are executed and presented in this section. The attitude-dynamics-related parameters are specified as follows: $I_{x x}=434270$ slug $\cdot \mathrm{ft}^{2}, I_{x z}=17880$ slug . $\mathrm{ft}^{2}, I_{y y}=961200 \operatorname{slug} \cdot \mathrm{ft}^{2}, I_{z x}=17880$ slug $\cdot \mathrm{ft}^{2}$ and $I_{z z}=1131541 \mathrm{slug} \cdot \mathrm{ft}^{2}$. Other elements that appear in I are set to zero. It is required that the system variables satisfy the constraints $\mathbb{X}_{\Theta}=\{\boldsymbol{\Theta}:\|\boldsymbol{\Theta}\| \leq 45 \mathrm{deg}\}, \mathbb{X}_{\omega}=$ $\{\boldsymbol{\omega}:\|\boldsymbol{\omega}\| \leq 5 \mathrm{deg} / \mathrm{s}\}$ and $\mathbb{U}_{M}=\left\{\mathbf{M}:\|\mathbf{M}\| \leq 1 \times 10^{5} \mathrm{lb} \cdot \mathrm{ft}\right\}$. We consider an attitude tracking scenario where the reference angular signals are given by $\alpha_{\mathrm{r}}=10+2.5 \sin (0.5 t), \beta_{\mathrm{r}}=0$, and $\sigma_{\mathrm{r}}=-30+5 \cos (0.5 t)$ respectively. The initial conditions for different variables are set as $\Theta(0)=[7.5,10,-30]^{T}$, $\boldsymbol{\omega}(0)=[0,0,0]^{T}$ and $\mathbf{M}(0)=[0,0,0]^{T}$. Hence, the initial feasibility is guaranteed. For the TRMPC algorithm, based on Lemma 2 and Lemma 4, the weight and local gain matrices are chosen as $\mathbf{P}=\operatorname{diag}\{8,8,8\}, \mathbf{Q}=\operatorname{diag}\{2,2,2\}$, $\mathbf{R}=\operatorname{diag}\{1,1,1\}$ and $\tilde{\mathbf{K}}=\operatorname{diag}\{2.5,2.5,2.5\}$ for the inner- and outer-loop TRMPC. $\boldsymbol{\Delta}_{f}$ and $\boldsymbol{\Delta}_{d}$ are bounded by $\eta_{1}=0.5$ and $\eta_{2}=0.1$. The feedback gain matrix is selected as $\mathbf{K}=\operatorname{diag}\{5,5,5\}$ for the two loops. The sampling period is set to $0.2 \mathrm{~s}$, while the prediction horizon $T=20 \mathrm{~s}$.

\section{B. Experimental Setup}

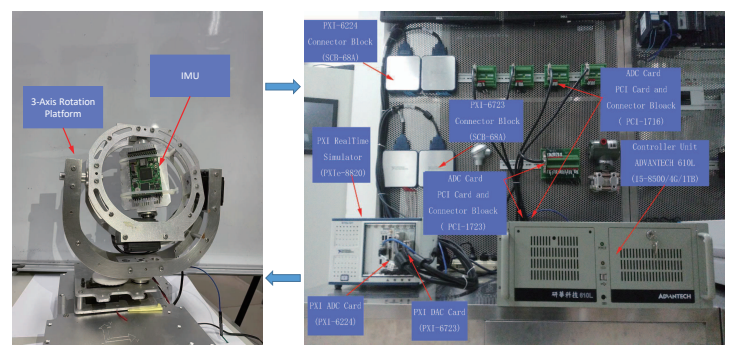

Fig. 3: System test platform

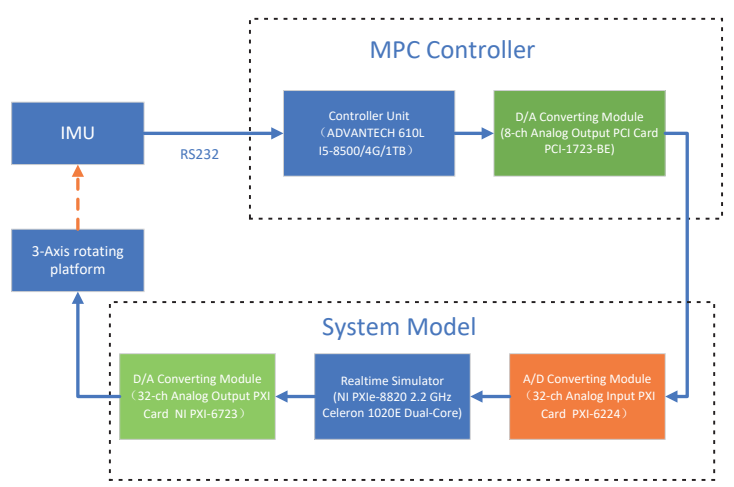

Fig. 4: Working principal of the platform

A hardware-in-the-loop platform is established and applied to perform the experimental tests. Specifically, as depicted in Fig. 3, the system test platform includes a spacecraft model simulator (NI PXIe-8820 2.2 GHz Celeron 1020E Dual-Core, NI PXI-6723 D/A converting module and PXI-6224 A/D converting module), a controller unit (ADVANTECH $610 \mathrm{~L}$ with I5-8500/4G/1TB and PCI-1723-BE D/A converting module), a 3-axis rotating platform and an inertial measurement unit (IMU). Fig. 4 illustrates the working principal of the platform. The spacecraft model, which considers the angular velocity 
and attitude angle dynamics, runs on the NI PXI real-time controller with NI LabVIEW real-time module version 8.0, which is used to drive the 3 -axis rotating platform by using a D/A converting module. The spacecraft parameters (angle of attack; sideslip and bank angles; and roll, pitch and yaw angular rates) obtained by the IMU are sent to the controller unit via RS232 and then employed to generate the proposed control law.

\section{Tracking Performance Evaluation and Comparative Studies}

The tracking performance achieved by applying the proposed dual-loop TRMPC algorithm in the presence of constraints and disturbances can be seen in Fig. 5. From this
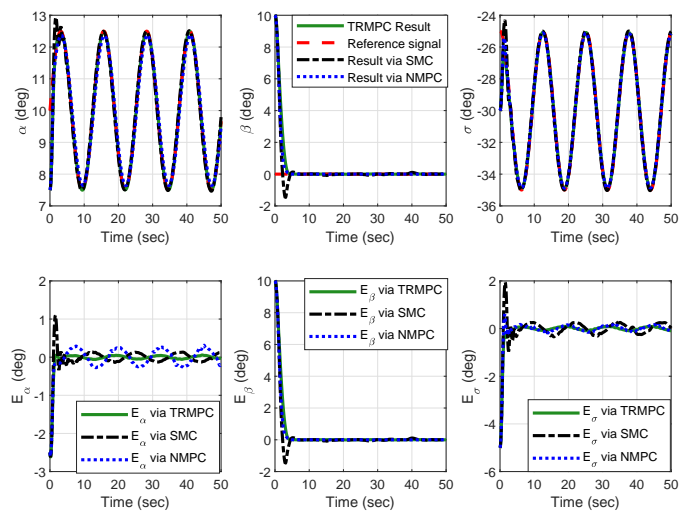

Fig. 5: Attitude tracking and attitude tracking error evolution

figure, it is obvious that the proposed approach is able to offer a promising tracking performance for the considered problem. That is, the desired angle profiles can be successfully tracked in a short time and the tracking errors of $\Theta$ can be rapidly steered to a small neighbourhood of the origin.
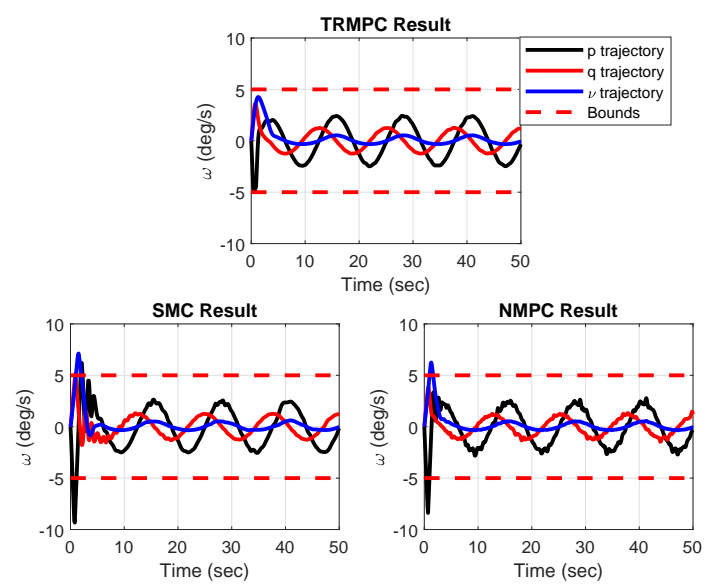

Fig. 6: Angular rate evolution trajectories

In Fig. 6 and Fig. 7, the corresponding angular rate evolution trajectories and the input profiles are visualized. It is clear from these two figures that all the variable constraints (indicated
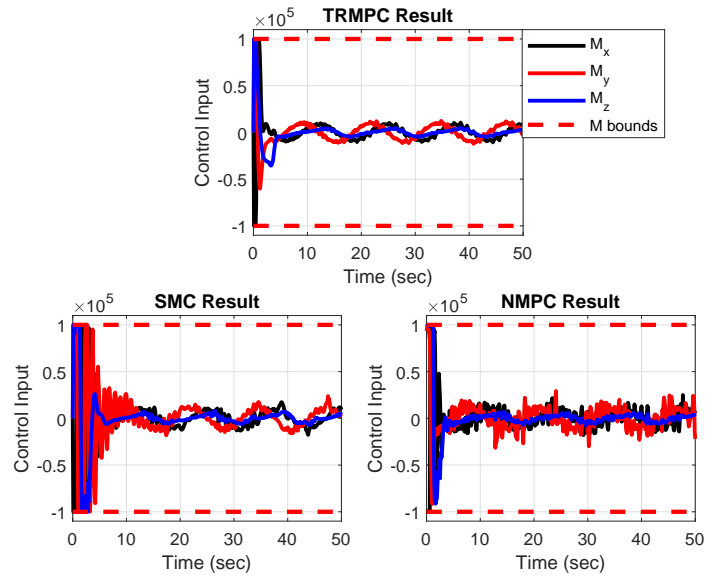

Fig. 7: Input evolution trajectories

by the red dashed line) are satisfied during the entire closedloop control process. Therefore, the effectiveness of restricting the nominal system state and input variables to a tightened region in the TRMPC optimization formulation is verified. Note that although some irregularity in the shape can be detected in the obtained angular rate profiles, the trajectory curves are relatively-smooth. Actually, the irregularity may caused by multiple reasons such as the consideration of additive disturbances acting on the angular rate dynamics, and the nonlinear and periodic characteristics of the reference angular trajectories. In addition, it is worth highlighting that by examining the input evolution profiles presented in Fig. 7, one can observe some small oscillations, which are mainly caused by the non-linear feedback part of the proposed control law. That is, the algorithm is trying to reduce the state deviations between the nominal system and the noise-perturbed system.

To highlight the advantages of applying TRMPC, comparative studies were performed between the proposed scheme and other newly developed approaches. The methods selected for comparison are the non-linear MPC (NMPC)-based attitude tracking method reported in [20] and the sliding mode control (SMC)-based tracking scheme proposed in [3]. Note that the selected NMPC can be viewed as a non-robust approach, as it ignores the disturbances in the dynamics and relies purely on the inherent robustness of deterministic MPC. The corresponding attitude angle tracking trajectories, angular rate and control input evolution profiles are depicted in Figs. 57. To quantitatively compare the reference tracking (steadystate) performances of different algorithms, the performance measure Ind $_{1}=\left(\int_{0}^{t_{f}}\left\|\mathbf{E}_{\theta}\right\|^{2} d t\right)^{\frac{1}{2}}$ is applied. Detailed results, including the scaled Ind $_{1}$ and the peak constraint values, are given in Table I for clear illustration.

TABLE I: Comparative results of different methods

\begin{tabular}{lcccc}
\hline \hline \multirow{2}{*}{ Method } & & \multicolumn{3}{c}{ Peak constraint } \\
\cline { 3 - 5 } & $\operatorname{Ind}_{1}$ & $\max (|p|)$ & $\max (|q|)$ & $\max (|\nu|)$ \\
\hline TRMPC & 131.0882 & 5.0000 & 3.6108 & 4.2630 \\
NMPC [20] & 137.4215 & 8.3778 & 3.7954 & 6.2475 \\
SMC [3] & 131.869 & 9.3319 & 5.1652 & 7.1271 \\
\hline \hline
\end{tabular}

According to the results shown in Figs. 5-7 and Table I, 
the proposed TRMPC method is able to achieve a better reference tracking performance than that of its counterparts (e.g., smaller Ind $_{1}$ value). In addition, harsher angular rate and control evolution profiles are obtained using the NMPCand SMC-based tracking approaches. More importantly, it can be seen from Fig. 6 that the NMPC- and SMC-based methods fail to maintain the actual angular rate profiles in the defined constraint regions due to the existence of additive disturbances. Therefore, we may conclude that the proposed TRMPC control scheme has advantages in terms of the physical constraints and disturbance rejection.

\section{Case Studies on Algorithm Parameters}

An important variable that requires further analysis is $\mathbb{O}_{\Theta}$. Its value reflects the variation between the controlled trajectory and the optimized nominal trajectory, i.e., with and without the robust feedback control part embedded in the proposed TRMPC. According to the TRMPC design, $\mathbb{O}_{\Theta}$ should be guaranteed to stay inside an invariant tube region. To better illustrate this point, the evolution trajectories of $\mathbb{O}_{\Theta}=\left[\alpha_{e}, \beta_{e}, \sigma_{e}\right]^{T}$ are visualized in Fig. 8. In addition, based on Lemma 3 , the invariant tube region can be expressed as

$$
\mathbb{O}_{\Theta}=\left\{\left[\alpha_{e}, \beta_{e}, \sigma_{e}\right]^{T} \in \mathbb{R}^{3} \mid\left[\begin{array}{l}
\left|\alpha_{e}\right| \\
\left|\beta_{e}\right| \\
\left|\sigma_{e}\right|
\end{array}\right] \leq\left[\begin{array}{c}
\frac{1}{10} \\
\frac{1}{10} \\
\frac{1}{10}
\end{array}\right]\right\}
$$
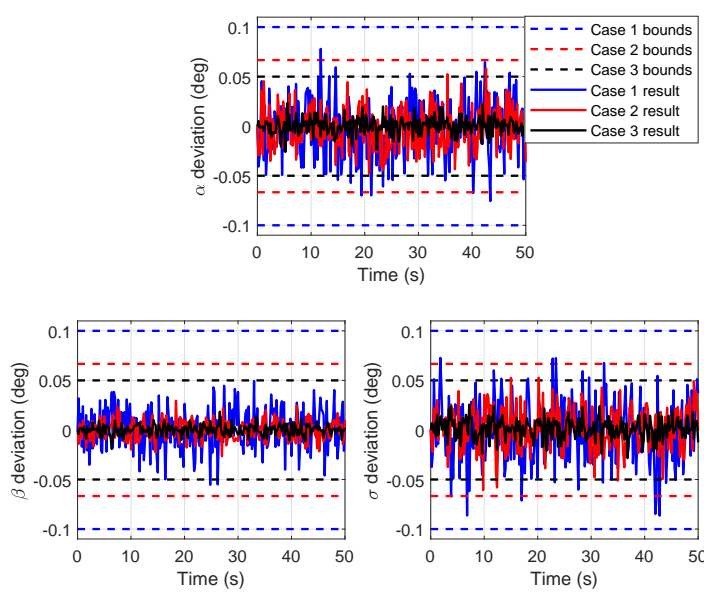

Fig. 8: Angular variable deviation trajectories

In Fig. 8, the tube boundaries are indicated by the blue dashed lines. From the result, it is obvious that the trajectories of $\alpha_{e}, \beta_{e}$, and $\sigma_{e}$ do not cross the boundary lines during the entire evolution. This outcome confirms that the proposed TRMPC is able to maintain the state deviation value in the invariant tube region.

Comparative case studies were performed to analyse the impact of selecting different gain matrices $\mathbf{K}$ on the tracking performance. Specifically, three cases are considered:

Case $1 \quad \mathbf{K}_{1}=\operatorname{diag}\{10,10,10\}$, resulting in $\mathbb{O}_{\Theta}^{1}=[0.1,0.1,0.1]^{T} ;$

Case $2 \mathbf{K}_{2}=\operatorname{diag}\{15,15,15\}$, resulting in $\mathbb{O}_{\Theta}^{2}=[0.067,0.067,0.067]^{T}$;

Case $3 \quad \mathbf{K}_{3}=\operatorname{diag}\{20,20,20\}$, resulting in $\mathbb{O}_{\Theta}^{3}=[0.05,0.05,0.05]^{T}$.

The corresponding state deviation results are visualized in Fig. 8, where the tube boundaries for Cases 1-3 are indicated by the blue, red and black dashed lines, respectively. Detailed tracking results are quantified and reported in Table II, where an additional indicator $\operatorname{Ind}_{2}=\left(\int_{0}^{t_{f}}\|\mathbf{M}\|^{2} d t\right)^{\frac{1}{2}}$ is introduced to measure the control cost. By viewing the state deviation

TABLE II: Results for different cases

\begin{tabular}{lcc}
\hline \hline \multirow{2}{*}{ Case No. } & \multicolumn{2}{c}{ Performance } \\
\cline { 2 - 3 } & Ind $_{1}$ & Ind $_{2}$ \\
\hline Case 1 & 131.0882 & 2.8285 \\
Case 2 & 129.7358 & 2.9950 \\
Case 3 & 119.1921 & 4.8494 \\
\hline \hline
\end{tabular}

curves shown in Fig. 8 and the results reported in Table II, we find that using a larger feedback gain value tends to result in a better angle tracking performance. Moreover, it can reduce the deviation between the actual state trajectories and the optimized nominal solutions. However, according to Lemma 3 , a larger feedback gain value will reduce the size of the state deviation tube, and because of this, more control efforts are likely to be consumed such that the actual state variable can be maintained in a relatively-tight tube region.

\section{CONCLUSIONS}

In this work, a dual-loop control framework capable of tracking the desired spacecraft attitude reference trajectory in the presence of disturbances and system constraints was established. To optimize the tracking performance while preserving robust constraint satisfaction, a new TRMPC algorithm was built and used as the controller to generate control actions for spacecraft angular and angular rate systems. In addition, through the design of a terminal control law and an invariant set, explicit conditions regarding the recursive feasibility and ISS were derived. Based on the obtained experimental results, it was verified that the proposed TRMPC is able to rapidly steer the attitude motion to track the desired reference trajectory without violating state and input constraints. Moreover, according to the comparative studies, the proposed TRMPC can achieve a better attitude tracking performance than that of other methods studied in this paper. Hence, we believe that TRMPC is a promising control algorithm and that it is reliable to apply the established attitude control scheme to deal with the considered reference tracking problem.

It is worth highlighting that the main shortcoming of the proposed TRMPC scheme is that due to the implementation of a robust feedback law, some degrees of conservativeness are introduced, thus degrading the solution optimality. Therefore, future research can be carried out to extend the TRMPC algorithm such that the conservativeness can be reduced while preserving system robustness.

\section{APPENDIX \\ PROOF OF THEOREM 2}

Proof. The entire proof contains two steps. First, we illustrate that the tracking error of the nominal attitude system can be steered to the origin. Subsequently, we illustrate that with application of the robust feedback law, the actual state trajectory can converge to an invariant tube region where the centre is the optimized trajectory of the nominal system. 
Specifically, for the outer attitude angle tracking error system, the optimal objective $J_{\theta}\left(\tilde{\mathbf{E}}_{\theta}^{*}\left(t_{k}\right), \tilde{\mathbf{U}}_{\theta}^{*}\left(t_{k}\right)\right)$ is chosen as the Lyapunov function. Then, the difference in this Lyapunov function $\Delta V_{\theta}$ at $t_{k}$ and $t_{k+1}$ satisfies the following inequality:

$$
\begin{aligned}
\Delta V_{\theta} \leq & J_{\theta}\left(\tilde{\mathbf{E}}_{\theta}\left(t_{k+1}\right), \tilde{\mathbf{U}}_{\theta}\left(t_{k+1}\right)\right)-J_{\theta}\left(\tilde{\mathbf{E}}_{\theta}^{*}\left(t_{k}\right), \tilde{\mathbf{U}}_{\theta}^{*}\left(t_{k}\right)\right) \\
= & -\int_{t_{k}}^{t_{k}+T}\left(\left\|\tilde{\mathbf{E}}_{\theta}^{*}\left(\tau \mid t_{k}\right)\right\|_{P}^{2}+\left\|\tilde{\mathbf{U}}_{\theta}^{*}\left(\tau \mid t_{k}\right)\right\|_{Q}^{2}\right) d \tau \\
& +\int_{t_{k+1}}^{t_{k+1}+T}\left(\left\|\tilde{\mathbf{E}}_{\theta}\left(\tau \mid t_{k+1}\right)\right\|_{P}^{2}+\left\|\tilde{\mathbf{U}}_{\theta}\left(\tau \mid t_{k+1}\right)\right\|_{Q}^{2}\right) d \tau \\
& -\left\|\tilde{\mathbf{E}}_{\theta}^{*}\left(t_{k}+T \mid t_{k}\right)\right\|_{R}^{2}+\left\|\tilde{\mathbf{E}}_{\theta}\left(t_{k+1}+T \mid t_{k+1}\right)\right\|_{R}^{2} \\
= & \int_{t_{k}+T}^{t_{k+1}+T}\left(\left\|\tilde{\mathbf{E}}_{\theta}\left(\tau \mid t_{k+1}\right)\right\|_{P}^{2}+\left\|\tilde{\mathbf{U}}_{\theta}\left(\tau \mid t_{k+1}\right)\right\|_{Q}^{2}\right) d \tau \\
& +\int_{t_{k+1}}^{t_{k}}\left(\left\|\tilde{\mathbf{E}}_{\theta}^{*}\left(\tau \mid t_{k}\right)\right\|_{P}^{2}+\left\|\tilde{\mathbf{U}}_{\theta}^{*}\left(\tau \mid t_{k}\right)\right\|_{Q}^{2}\right) d \tau \\
& -\left\|\tilde{\mathbf{E}}_{\theta}^{*}\left(t_{k}+T \mid t_{k}\right)\right\|_{R}^{2}+\left\|\tilde{\mathbf{E}}_{\theta}\left(t_{k+1}+T \mid t_{k+1}\right)\right\|_{R}^{2}
\end{aligned}
$$

In (38), $J_{\theta}\left(\tilde{\mathbf{E}}_{\theta}\left(t_{k}\right), \tilde{\mathbf{U}}_{\theta}\left(t_{k}\right)\right)$ represents the objective value of the constructed solution (37). Note that in Lemma 2, we showed that $\dot{g}_{\theta}\left(\tilde{\mathbf{E}}_{\theta}\left(\tau \mid t_{k}\right)\right)+L_{\theta}\left(\tilde{\mathbf{E}}_{\theta}\left(\tau \mid t_{k}\right), \tilde{\mathbf{U}}_{\theta}\left(\tau \mid t_{k}\right)\right) \leq 0$. Integrating this equation from $t_{k}+T$ to $t_{k+1}+T$ and substituting the outcome into (38), one can obtain $\Delta V_{\theta} \leq 0$. Hence, the angle tracking error vector $\tilde{\mathbf{E}}_{\theta}$ will asymptotically converge to the origin, and because of this, a $\mathcal{K}$ function exists such that for any $t>0$, we have $\left\|\tilde{\mathbf{E}}^{*}(t)\right\| \leq \mathcal{K}\left(\mathbf{E}^{*}(0), t\right)$.

According to Lemma 3 (2) and Theorem 1, we can obtain $O_{\theta}(t) \in \mathbb{O}_{\theta}$, which means there exists a $\mathcal{K}_{\infty}$ function such that for any $t>0,\left\|O_{\theta}(t)\right\| \leq \mathcal{K}_{\infty}\left(\eta_{1}\right)$. Consequently, it is obvious that for any $t>0$, one has $\|\mathbf{E}(t)\| \leq \mathcal{K}\left(\mathbf{E}^{*}(0), t\right)+$ $\mathcal{K}_{\infty}\left(\eta_{1}\right)$, implying that the actual attitude angle tracking error is ultimately bounded and that the outer tracking error system has ISS. The proof for the inner tracking system follows the same procedure and is thus omitted here.

\section{References}

[1] S. Yin, B. Xiao, S. X. Ding, and D. Zhou, "A review on recent development of spacecraft attitude fault tolerant control system," IEEE Transactions on Industrial Electronics, vol. 63, no. 5, pp. 3311-3320, 2016.

[2] R. Chai, A. Savvaris, A. Tsourdos, S. Chai, and Y. Xia, "Trajectory optimization of space maneuver vehicle using a hybrid optimal control solver," IEEE Transactions on Cybernetics, vol. 49, no. 2, pp. 467-480, 2019.

[3] J. Qiao, Z. Li, J. Xu, and X. Yu, "Composite nonsingular terminal sliding mode attitude controller for spacecraft with actuator dynamics under matched and mismatched disturbances," IEEE Transactions on Industrial Informatics, vol. 16, no. 2, pp. 1153-1162, 2020.

[4] L. Cao, B. Xiao, M. Golestani, and D. Ran, "Faster fixed-time control of flexible spacecraft attitude stabilization," IEEE Transactions on Industrial Informatics, vol. 16, no. 2, pp. 1281-1290, 2020.

[5] L. Sun and Z. Zheng, "Saturated adaptive hierarchical fuzzy attitudetracking control of rigid spacecraft with modeling and measurement uncertainties," IEEE Transactions on Industrial Electronics, vol. 66, no. 5, pp. 3742-3751, 2019.

[6] Q. Mao, L. Dou, Z. Yang, B. Tian, and Q. Zong, "Fuzzy disturbance observer-based adaptive sliding mode control for reusable launch vehicles with aeroservoelastic characteristic," IEEE Transactions on Industrial Informatics, vol. 16, no. 2, pp. 1214-1223, 2020.

[7] B. Tian, L. Yin, and H. Wang, "Finite-time reentry attitude control based on adaptive multivariable disturbance compensation," IEEE Transactions on Industrial Electronics, vol. 62, no. 9, pp. 5889-5898, 2015.

[8] B. Cui, Y. Xia, K. Liu, Y. Wang, and D. Zhai, "Velocity-observer-based distributed finite-time attitude tracking control for multiple uncertain rigid spacecraft," IEEE Transactions on Industrial Informatics, vol. 16, no. 4, pp. 2509-2519, 2020.
[9] F. Bayat, "Model predictive sliding control for finite-time three-axis spacecraft attitude tracking," IEEE Transactions on Industrial Electronics, vol. 66, no. 10, pp. 7986-7996, 2019.

[10] D. Limon, A. Ferramosca, I. Alvarado, and T. Alamo, "Nonlinear mpc for tracking piece-wise constant reference signals," IEEE Transactions on Automatic Control, vol. 63, no. 11, pp. 3735-3750, 2018.

[11] C. Shen, Y. Shi, and B. Buckham, "Trajectory tracking control of an autonomous underwater vehicle using lyapunov-based model predictive control," IEEE Transactions on Industrial Electronics, vol. 65, no. 7 , pp. 5796-5805, 2018.

[12] Ø. Hegrenæs, J. T. Gravdahl, and P. Tøndel, "Spacecraft attitude control using explicit model predictive control," Automatica, vol. 41, no. 12, pp. 2107-2114, 2005.

[13] A. Weiss, M. Baldwin, R. S. Erwin, and I. Kolmanovsky, "Model predictive control for spacecraft rendezvous and docking: Strategies for handling constraints and case studies," IEEE Transactions on Control Systems Technology, vol. 23, no. 4, pp. 1638-1647, 2015.

[14] A. Guiggiani, I. Kolmanovsky, P. Patrinos, and A. Bemporad, "Fixedpoint constrained model predictive control of spacecraft attitude," in 2015 American Control Conference (ACC), 2015, Conference Proceedings, pp. 2317-2322.

[15] A. Altan and R. Hacioğlu, "Modeling of three-axis gimbal system on unmanned air vehicle (uav) under external disturbances," in 2017 25th Signal Processing and Communications Applications Conference (SIU), Conference Proceedings, pp. 1-4.

[16] A. Altan, A. Ö, and R. Hacioğlu, "Real-time control based on narx neural network of hexarotor uav with load transporting system for path tracking," in 2018 6th International Conference on Control Engineering \& Information Technology (CEIT), Conference Proceedings, pp. 1-6.

[17] A. Altan and R. Hacıoğlu, "Hammerstein model performance of three axes gimbal system on unmanned aerial vehicle (uav) for route tracking," in 2018 26th Signal Processing and Communications Applications Conference (SIU), Conference Proceedings, pp. 1-4.

[18] _ - "Model predictive control of three-axis gimbal system mounted on uav for real-time target tracking under external disturbances," $M e$ chanical Systems and Signal Processing, vol. 138, p. 106548, 2020.

[19] A. Altan, "Performance of metaheuristic optimization algorithms based on swarm intelligence in attitude and altitude control of unmanned aerial vehicle for path following," in 2020 4th International Symposium on Multidisciplinary Studies and Innovative Technologies (ISMSIT), Conference Proceedings, pp. 1-6.

[20] H. Li, W. Yan, and Y. Shi, "Continuous-time model predictive control of under-actuated spacecraft with bounded control torques," Automatica, vol. 75, pp. 144-153, 2017.

[21] L. Magni, G. De Nicolao, R. Scattolini, and F. Allgöwer, "Robust model predictive control for nonlinear discrete-time systems," International Journal of Robust and Nonlinear Control, vol. 13, no. 3-4, pp. 229246, 2003.

[22] D. M. Raimondo, D. Limon, M. Lazar, L. Magni, and E. F. Camacho, "Min-max model predictive control of nonlinear systems: A unifying overview on stability," European Journal of Control, vol. 15, no. 1, pp. $5-21,2009$.

[23] D. Q. Mayne, E. C. Kerrigan, E. J. van Wyk, and P. Falugi, "Tubebased robust nonlinear model predictive control," International Journal of Robust and Nonlinear Control, vol. 21, no. 11, pp. 1341-1353, 2011.

[24] S. Yu, C. Maier, H. Chen, and F. Allgöwer, "Tube mpc scheme based on robust control invariant set with application to lipschitz nonlinear systems," Systems \& Control Letters, vol. 62, no. 2, pp. 194-200, 2013.

[25] R. Chai, A. Tsourdos, A. Savvaris, Y. Xia, and S. Chai, "Real-time reentry trajectory planning of hypersonic vehicles: A two-step strategy incorporating fuzzy multiobjective transcription and deep neural network," IEEE Transactions on Industrial Electronics, vol. 67, no. 8, pp. 6904-6915, 2020.

[26] B. Tian, W. Fan, R. Su, and Q. Zong, "Real-time trajectory and attitude coordination control for reusable launch vehicle in reentry phase," IEEE Transactions on Industrial Electronics, vol. 62, no. 3, pp. 1639-1650, 2015.

[27] J. Fleming, B. Kouvaritakis, and M. Cannon, "Robust tube mpc for linear systems with multiplicative uncertainty," IEEE Transactions on Automatic Control, vol. 60, no. 4, pp. 1087-1092, 2015.

[28] D. Muñoz Carpintero, B. Kouvaritakis, and M. Cannon, "Striped parameterized tube model predictive control," Automatica, vol. 67, pp. 303$309,2016$. 


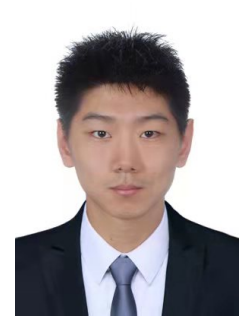

Runqi Chai (Member, IEEE) received the B.S. degree in information and computing science from the North China University of Technology, Beijing, China, in 2015 and the Ph.D. degree in Aerospace Engineering from Cranfield University, Cranfield, U.K, in August 2018. He is currently a research fellow at Cranfield University. His research interests include trajectory optimization, networked control systems, and multiagent control systems.

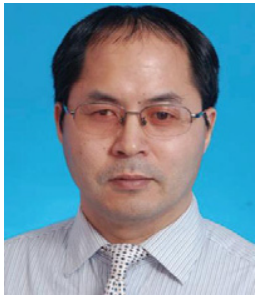

Yuanqing Xia (Senior Member, IEEE) was born in Anhui Province, China, in 1971. He received the B.S. degree in fundamental mathematics from the Department of Mathematics, Chuzhou University, Chuzhou, China, in 1991, the M.S. degree in fundamental mathematics from Anhui University, Wuhu, China, in 1998, and the Ph.D. degree in control theory and control engineering from the Beijing University of Aeronautics and Astronautics, Beijing, China, in 2001. His current research interests are in the fields of networked control systems, robust control and signal processing, active disturbance rejection control and flight control. He has published 8 monographs with Springer and Wiley, and more than 300 papers in journals. He has obtained Second Award of the Beijing Municipal Science and Technology (No. 1) in 2010, Second National Award for Science and Technology (No. 2) in 2011, and Second Natural Science Award of The Ministry of Education (No. 1) in 2012. He is a Deputy Editor of the Journal of the Beijing Institute of Technology, Associate Editor of Acta Automatica Sinica, Control Theory and Applications, the International Journal of Innovative Computing, Information and Control, and the International Journal of Automation and Computing.

Antonios Tsourdos (Member, IEEE) obtained a MEng on Electronic, Control and Systems Engineering from the University of Sheffield, in 1995, an MSc on Systems Engineering from Cardiff University in 1996 and a PhD on Nonlinear Robust Autopilot Design and Analysis from Cranfield University in 1999. He joined the Cranfield University in 1999 as lecturer, appointed Head of the Centre of Autonomous and Cyber-Physical Systems in 2007 and Professor of Autonomous Systems and Control in 2009 and Director of Research - Aerospace, Transport and Manufacturing in 2015. He leads the research theme on autonomous systems within the School of Aerospace, Transport and Manufacturing at Cranfield University. He has diverse expertise in both unmanned and autonomous vehicles as well as networked systems. He conducts basic and applied research in the fields of guidance, control and navigation for single and multiple unmanned autonomous vehicles as well as research on cyber-physical systems.

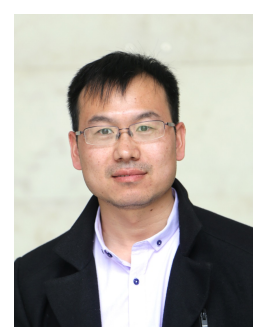

Senchun Chai (Senior Member, IEEE) received the B.S. and master's degrees in control science and engineering from Beijing Institute of Technology, Beijing, China in 2001 and 2004 , respectively. He received the Ph.D. degree in Networked Control System from University of South Wales, Pontypridd, U.K., in 2007.

$\mathrm{He}$ is currently a professor of School of Automation with Beijing Institute of Technology. He was a research fellow at Cranfield University, UK, from 2009 to 2010, and was a visiting scholar at University of Illinois at Urbana-Champaign Urbana, USA, from January 2010 to May 2010. He has published over 100 journal and conference papers. His current research interests focus on flight control system, networked control systems, embedded systems and multi-agent control systems.

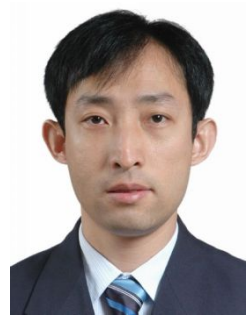

Huijun Gao (Fellow, IEEE) received the Ph.D. degree in control science and engineering from the Harbin Institute of Technology, Harbin, China. He was a Research Associate with the Department of Mechanical Engineering, The University of Hong Kong, Hong Kong, from 2003 to 2004 . He held a post-doctoral research position with the Department of Electrical and Computer Engineering, University of Alberta, Edmonton, AB, Canada, from 2005 to 2007. He has been with the Harbin Institute of Technology since 2004, where he is currently a Professor and the Director of the Research Institute of Intelligent Control and Systems. His current research interests include network-based control, robust control and filtering, and their engineering applications. Prof. Gao is an Administrative Committee Member of the IEEE Industrial Electronics Society. He is an Associate Editor of Automatica, the IEEE Transactions on Control Systems Technology, the IEEE Transactions on Cybernetics, and the IEEE/ASME Transactions on Mechatronics. 


\section{Dual-loop tube-based robust model predictive attitude tracking control for spacecraft with system constraints and additive disturbances}

Chai, Runqi

IEEE

Chai R, Tsourdos A, Gao H, et al., (2022) Dual-loop tube-based robust model predictive attitude tracking control for spacecraft with system constraints and additive disturbances. IEEE Transactions on Industrial Electronics, Volume 69, Number 4, April 2022, pp. 4022-4033 https://doi.org/10.1109/TIE.2021.3076729 Downloaded from Cranfield Library Services E-Repository 